\title{
Student Model-based Adapted e-Learning
}

\author{
Shehab Gamalel-Din \\ Al-Azhar University, Computer \& Systems Engineering \\ drshehabg@yahoo.com
}

It is becoming evident that educated and skilled human resources and workers are real assets and a key of success and power for both nations and organizations. Therefore, education and training play an important role in the competing world. However, several challenges exist; among them are:

- Availability of educational opportunities and resources to cope with the increased demand due to expanding population.

- Shortage in resources: space, budgetary, qualified instructors/teachers/ professors, educational material and resources ... etc.

- Continuous demand on retraining and conversion training to deal with the increased rate of obsolescence due to the continuous evolution in technology and work practices.

- Quality education and employees training to withstand the tough competition in the globalization age that mandates an increased need for highly skilled employees.

- Expanding training opportunities for employees of the same global organization at geographically dispersed locations with different cultures and backgrounds. Decreasing educational cost while maintaining high quality.

Students, parents, and educators are increasingly unhappy with the traditional classroom model that has continued to rely upon a "one size fits all" paradigm and that does not adequately address the individual students' learning preferences. They are opt of replacing that system with one where instruction is individualized to students' unique needs.

Although multimedia learning material and Computer-Based Training (CBT) offered a solution to some of the above challenges, however, they are still lacking an essential component for effective teaching, namely personalizing and adapting the delivery of a course in terms of the student profile (student model) as the human expert teacher does. 
In traditional classrooms, especially in tutoring sessions with small groups of students, the human teacher utilizes his experience and intelligence to adapt his/her teaching method and style to meet the average student in the classroom. It is well known that as small the learning group as the more effective the learning is. This is because each individual student can be considered unique in his/her learning profile. One can say 'there can be as many learning profiles as there are students. Students differ in their learning goals, in their style of perceiving new knowledge, in their memorization vs. analytical attitudes towards new knowledge, in their way of relating new knowledge to known facts, in their problem-solving model, ... etc.

Understanding the needs of each individual student and offering him/her personal instruction helps equip the learner with everything he/she needs to learn successfully. Therefore, the most effective and efficient learning system is the oneto-one tutoring where the instructor understands the learning profile of his/her sole student and tailors his/her teaching strategy and pedagogy accordingly.

Intelligent adaptive e-Learning environments introduce one possible solution to many of the above challenges. We have recognized this hypothesis early this millennium, and therefore focused our research on modeling and designing intelligent e-Learning systems with a hope to draw a picture of the future of the electronic education system. Over the years and along this research dimension, we came with a model and a prototype of how the Smart School of the future will look like $[1,2]$.

In this paper, we focus on one angle of our bigger picture of research, namely, adaptive/personalized Learning environments. We have developed a unique model of adaptive learning; called it the "Smart Adaptive e-Learning Model" (SAeLM). We have also developed, tested, and experimented a prototype.

Intelligent adaptive learning is a new class of education technology that captures every decision a student makes and adjusts the student's learning paths both within lessons and between lessons, thereby providing millions of personalized learning paths, each tailored to a student's unique needs in real time. However, SAeLM offers more than just adapting the learning paths and strategies, in addition, it 
adapts the content presentation and the interaction interfaces with the student in such a way suiting his/her preferred style.

The SAeLM environment is a whole suite of products that support the industry of education/training in many directions to serve all the stakeholders of the learning process, such as teachers, students, and education administrators, as shown in Figure 1. The focus was on adaptive learning in a unique approach that employs multidisciplinary techniques and theories, e.g., AI, psychometrics, cognitive science, instructional design theories, etc. The suite of tools offers tools for an intelligent and automatic course authoring, adaptive course delivery, and a Smart adaptive e-Textbook. The proposed solution serves many of the contemporary education systems, such as flipped classes, blended education, and self-pace eLearning. The authoring tools automatically generate the course syllabus, teaching strategy, and e-textbook out of the instructor's defined course learning objectives; all are adapted to the student preferred style and knowledge/skills status quo, in addition to many other diversifying parameters.

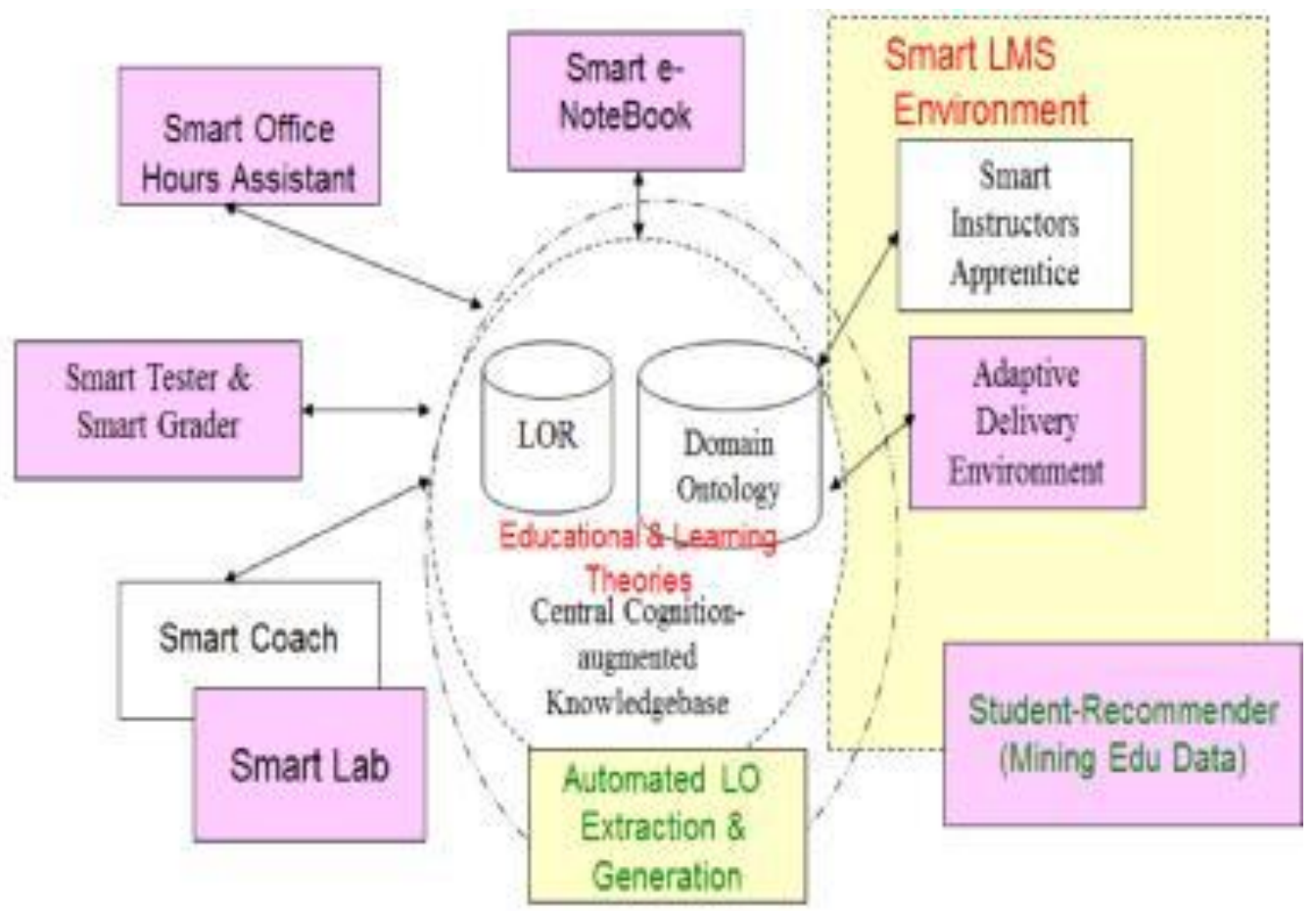

Figure 1. The Smart Virtual School of the Future platform. 
SAeLM centers its adaptation process around the student model, specifically, his/her background knowledge, and cognitive knowledge, namely, his/her learning style and IQ index. Experiments conducted on the developed prototype revealed improvements in the efficiency (study time) of an average of $30 \%$, and efficacy (learning effectiveness) improvement of $20 \%$ on the average [1, 2, 3, 4].

In fact, intelligent adaptive e-Learning is a paradigm shift in education through:

Empowering students for smarter and more effective learning ability. Empowering teachers for smarter course preparation and delivery.

The SAeLM prototype offers three adaptive components:

In this project, the focus is only on $\mathbf{S}^{\mathbf{3}}$ components that are in support of adaptive learning:

1. Smart Authoring Apprentice,

2. Smart e-Tutor, and

3. Smart e-TextBook.

The Smart e-TextBook is not a textbook as the word usually connotes, but rather it is a personalized course material that is carefully selected and properly ordered and presented to the student either online (via Cloud) or offline (on the student's laptop/tablet). The intention is to replace the current practice of one-book-fits-allstudents with a new concept of one-for-one e-Text book as depicted by Figure 2.

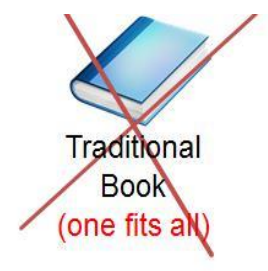

A personalized edition for each student

1. Background Knowledge

2. Learning Style

3. IQ

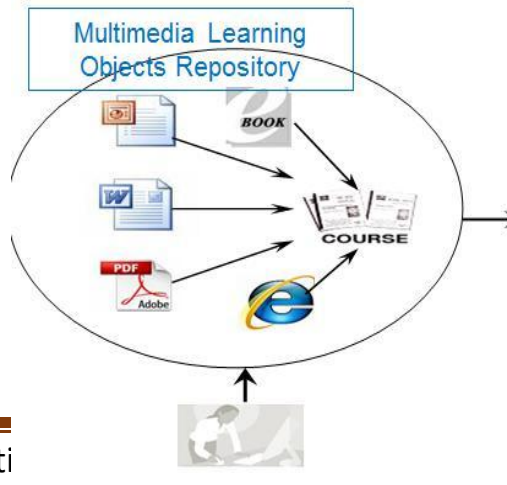

Internati 
Figure 2. Eliminating the One-Fits-All Textbook in favor of Personalized e-TextBook- One Edition for each Student.

The Smart e-TextBook system generates, as many personalized editions of Smart $e$-TextBooks as the number of students, one for each individual student that is especially personalized to meet his/her own personal student model. In other words, each student will have his/her personalized edition of the textbook (MySmart e-TextBook) that is usually different from those of his/her colleagues. It is worth noting that all the generated editions fulfil the same Course Learning Objectives (CLOs), but with adapted teaching strategy and pedagogy according to each individual owner student's profile; specifically his/her background knowledge and skills, learning style, IQ, age, education level, cultural background, ...etc.

The Smart Authoring (SA) system is a totally new approach in course authoring. It automates the authoring process completely. Once the CLOs are defined by the educational institution (and in some cases by mature trainees themselves), it takes the process from there and intelligently generates the adapted courseware that is completely personalized for one of two users, namely, the student and the teacher. In order to do its task, all what it needs are:

1. A Smart Domain Knowledge Ontology which is a specially designed semantic net that describes the domain concepts and the relationships tying them together, and

2. A Learning Objects Repository (LOR) hosting LOs that are described in a special set of metadata attributes.

The Smart e-Tutor is a learning delivery system that delivers the uniquely generated learning course as defined by the Smart authoring system and the Smart e-TextBook. It offers the student a friendly UI. In its delivery, it also intelligently adapts the UI according to the student profile (e.g., Learning Style). Another adaptivity dimension is the dynamic maneuvering through the course learning 
paths according to the student performance and his/her achieved knowledge/skill mastering levels during delivery time.

References:

1. Shehab Gamalel-Din, "Smart E-Learning School of the Future: Project Report", In the

Proceedings of the $6^{\text {th }}$ E-learning Applications Conference, Cairo, $10-12$ Jan, 2009.

2. Shehab Gamalel-Din, "Smart e-Learning: A greater perspective; from the fourth to the fifth generation e-learning", Egyptian Informatics Journal EIJ (sponsored by Elsevier), Vol. 11, No. 1, June 2010, pp. 39-48.

3. Shehab Gamalel-Din, and Reem Al-Otaibi, "Smart Assistant for Adaptive Course Preparation and Delivery in E-Learning Environments", In the Proceedings of the $7^{\text {th }}$ European Conference on e-Learning, Cyprus, 6-7 November 2008.

4. Reem Al-Otaibi, and Shehab Gamalel-Din, "Intelligent Querying For Adaptive Course Preparation and Delivery in E-Learning", In the Proceedings of the Ninth IASTED International Conference on Webbased Education, Sharm El-Sheikh, 15-17 March, 2010. 